\title{
In-depth phenotyping of a Donnai-Barrow patient helps clarify proximal tubule dysfunction
}

\author{
Angélique Dachy • François Paquot • Guillaume Debray • \\ Christophe Bovy • Erik I. Christensen • Laure Collard • \\ François Jouret
}

Received: 29 October 2014 / Revised: 11 December 2014 / Accepted: 16 December 2014 /Published online: 31 March 2015

(C) IPNA 2015

\begin{abstract}
Background The megalin/cubilin/amnionless complex is essential for albumin and low molecular weight (LMW) protein reabsorption by renal proximal tubules (PT). Mutations of the $L R P 2$ gene encoding megalin cause autosomal recessive Donnai-Barrow/facio-oculo-acoustico-renal syndrome (DB/ FOAR), which is characterized by LMW proteinuria. The pathophysiology of DB/FOAR-associated PT dysfunction remains unclear.

Clinical case A 3-year-old girl presented with growth retardation and proteinuria. Clinical examination was unremarkable, except for a still-opened anterior fontanel and myopia. Psychomotor development was delayed. At 6, she developed sensorineural hearing loss. Hypertelorism was noted when she turned 12. Blood analyses, including renal function parameters, were normal. Urine sediment was bland. Proteinuria was significant and included albumin and LMW proteins. Immunoblotting analyses detected cubilin and type 3 carbonic anhydrase (CA3) in the urine. Renal ultrasound was unremarkable. Optical examination of a renal biopsy did not
\end{abstract}

\footnotetext{
A. Dachy $\cdot$ L. Collard

Division of Pediatrics, CHC Liège, Liège, Belgium

F. Paquot $\cdot$ C. Bovy $\cdot$ F. Jouret

Division of Nephrology, University of Liège Hospital (Ulg CHU),

Liège, Belgium

G. Debray

Division of Genetics, University of Liège Hospital (Ulg CHU),

Liège, Belgium

E. I. Christensen

Department of Biomedicine, University of Aarhus, Aarhus, Denmark

L. Collard $(\bowtie)$

CHC Clinique de l'Espérance, Rue Saint Nicolas 447, B-4420,

Montegnée, Belgium

e-mail: laurecollard@gmail.com
}

disclose any tubular or glomerular abnormality. Electron microscopy revealed that PT apical endocytic apparatus was significantly less developed. Immunostaining for megalin showed a faint signal in PT cytosol contrasting with the distribution of cubilin at the apical membrane. The diagnostic procedure led to identifying two mutations of the $L R P 2$ gene. Conclusions The functional loss of megalin in DB/FOAR causes PT dysfunction characterized by increased urinary shedding of $\mathrm{CA} 3$ and cubilin.

Keywords Facio-oculo-acoustico-renal syndrome · Low-molecular-weight proteins $\cdot$ Receptor-mediated endocytosis · Megalin · Cubilin · Type 3 carbonic anhydrase

\section{Introduction}

Plasma albumin and low molecular weight (LMW) proteins are continuously filtered through renal glomeruli and reabsorbed by epithelial cells lining the proximal tubules (PT) via receptor-mediated endocytosis (RME). In PT, RME implicates the megalin/cubilin/amnionless complex [1]. Megalin is a multi-ligand receptor encoded by the $L R P 2$ gene, and belongs to the low-density lipoprotein (LDL) receptor family [2]. Mutations of $L R P 2$ cause autosomal recessive Donnai-Barrow/facio-oculo-acoustico-renal syndrome (DB/ FOAR), whose cardinal features include: corpus callosum agenesis, developmental delay, enlarged anterior fontanel, myopia, hypertelorism, sensorineural hearing loss, and LMW proteinuria [2, 3]. Megalin-null mice also demonstrate LMW proteinuria, which emphasizes the role of megalin in PT RME [4]. Megalin cooperates with another receptor, named cubilin, which associates with its partner, amnionless (AMN). At the apical pole of PT epithelial cells, ligand 
binding to the megalin/cubilin/AMN complex induces internalization into coated vesicles and delivery to endosomes and lysosomes for ligand processing and receptor degradation or recycling [5]. Progression along the endocytic apparatus requires vesicular acidification, which is driven by the electrogenic vacuolar $\mathrm{H}^{+}$-ATPase and a neutralizing $\mathrm{Cl}^{-}$conductance [6]. The loss of such a $\mathrm{Cl}^{-}$conductance leads to RME dysfunction in PT, like the one observed in Dent's disease [5, 7]. Dent's disease is an X-linked renal tubulopathy characterized by LMW proteinuria, hypercalciuria, and renal failure. It is caused by mutations in the CLCN5 gene encoding the $\mathrm{Cl}^{-} /$ $\mathrm{H}^{+}$exchanger, ClC-5 [5]. Similarly to Dent's disease, genetic inactivation of Clcn5 in mouse induces a severe trafficking defect in PT cells, with loss of megalin and cubilin at the brush border and LMW proteinuria [7]. Furthermore, ClC-5 inactivation causes urinary excretion of type 3 carbonic anhydrase (CAIII) [8]. CAIII has been uniquely detected in the urine of ClC-5- or megalin-deficient mice and patients with Dent's disease. However, data are lacking regarding CAIII excretion in the urine of megalin-deficient patients. We report on a girl presenting with typical DB/FOAR. On the basis of a 10 -year follow-up, repeated urine and blood samples, kidney biopsy materials, and genetic testing, we further characterize the genotype, phenotype, and pathophysiology of this rare entity.

\section{Clinical case}

A 3-year-old girl was evaluated for growth retardation and proteinuria. She was born at 38 gestational weeks. Growth retardation was initially linked to heavy maternal smoking during pregnancy. Her parents were non-consanguineous. Her half-brothers were healthy. Clinical examination was unremarkable, except for a still-opened anterior fontanel (which closed at 4) and bilateral myopia. Psychomotor development was delayed, which prompted the institutionalization of the child. At the age of 6 , she developed bilateral sensorineural hearing loss. Hypertelorism was noted when she turned 12.

Blood analyses, including hemoglobin, creatinine, $\mathrm{Na}^{+}$, $\mathrm{Cl}^{-}, \mathrm{K}^{+}$, albumin, uric acid, $25(\mathrm{OH})$ and $1,25(\mathrm{OH})$ vitamin $\mathrm{D}$ and thyroid hormones, were within the normal ranges, except for low levels of transthyretin. Urine sediment was bland. Heavy proteinuria $(1.990 \mathrm{mg} / \mathrm{g}$ creatinine) was found, including albumin $(290 \mathrm{mg} / \mathrm{g}$ creatinine) and LMW proteins $(\alpha 1$ microglobulin, $522 \mathrm{mg} / \mathrm{g}$ creatinine; retinol-binding protein (RBP), $215 \mathrm{mg} / \mathrm{g}$ creatinine; neutrophil gelatinase-associated lipocalin (NGAL), $14 \mathrm{IU} / \mathrm{l}$; transferrin, $55 \mathrm{mg} / \mathrm{l}$; cystatin, $61 \mathrm{mg} / \mathrm{l}$ ). Immunoblotting analyses also detected cubilin and CAIII in the urine (Fig. 1a, b). Kidney ultrasound was unremarkable. A renal biopsy was performed and examined by both light and electron microscopy. Optical examination did not disclose any tubular or glomerular abnormality. Electron microscopy revealed that PT apical endocytic apparatus was
Fig. 1 a, b Immunoblotting analyses of urine samples from a patient with Donnai-Barrow/facio-oculo-acoustico-renal syndrome (DB/FOAR). a Urinary proteins (equivalent to $10 \mu \mathrm{g}$ of creatinine in all lanes) of a healthy control, a patient with DB/FOAR syndrome and a patient with Dent's disease were separated by SDS-PAGE and visualized after Coomassie Blue staining. LMWP low molecular weight proteins. b Similar urinary samples were separated by SDS-PAGE, transferred onto a nitrocellulose membrane and blotted with antibodies against cubilin (from Ref. [3]), type 3 carbonic anhydrase (CAIII, from Ref. [8]) or Tamm-Horsfall protein (UMOD, from Ref. [15]). c Electron microscopy of renal proximal tubule cells from a patient with DB/ FOAR. Renal tissue samples were collected from the patient for diagnostic purposes, fixed in glutaraldehyde, and embedded in Epon. Ultrathin slides were prepared using Leica Reichert Ultracut S, and visualized on a Zeiss LE0909 electron microscope. The ultrastructure of PT cells appears normal, except for the apical endocytic apparatus (white frame). The number of coated pits, early endosomes, and dense apical tubules is decreased (inset). The brush border looks regular. d Immunostaining analysis of kidney samples from a patient with DB/ FOAR. Renal tissue samples were fixed in 4\% paraformaldehyde and embedded in paraffin. Serial sections (as notified by the asterisk located in the same renal tubule) were incubated with primary antibodies against megalin (from Ref. [3]), aquaporin-1 (from Ref. [15]), cubilin (from Ref. [3]) or type III carbonic anhydrase (CAIII, from Ref. [8]), followed by incubation with ad hoc horseradish-conjugated secondary antibodies. Images were taken using a Leica MC170 HD camera installed on a Leica DM1000 LED microscope. The typical distribution of megalin, aquaporin-1, and cubilin at PT brush border (arrowheads) is shown in the upper panels. In the lower panels, arrowheads indicate the apical distribution of cubilin at the brush border of proximal tubules (PT), which are identified by the co-expression of the water channel, aquaporin-1. Megalin is faintly detected in PT cytosol. CAIII is only detected in some PT cells (PT + ), whereas no signal is detected in others (PT-). Such a dispersed distribution along the PT was observed in kidneys from both ClC-5-deficient mice and patients with Dent's disease [8]. CAIII may participate in PT cell response to oxidative damage

significantly less developed, with a decreased number of coated pits, early endosomes, and dense apical tubules (Fig. 1c). Immunostaining for megalin showed a faint and diffuse signal in PT cells contrasting with the apical distribution of cubilin (Fig. 1d). CAIII was detected in the cytosol of some PT cells (Fig. 1d).

The diagnostic procedure led to sequencing the $L R P 2$ gene. Two mutations were identified: C.7564T $>$ C (p.Y2522H) and c. $12623 \mathrm{C}>\mathrm{A}$ (p.P4208H). Clinical and genetic testing of the parents could not be performed for ethical reasons. In the context of heavy mixed proteinuria, treatment initially included angiotensin-converting enzyme inhibitors and indomethacin. This treatment had no impact on proteinuria, and was stopped after a few months. At the age of 12 , renal function of the patient was normal, despite constant proteinuria.

\section{Discussion}

The patient presents with most of the cardinal features of DB/FOAR, including urinary loss of albumin and LMW 


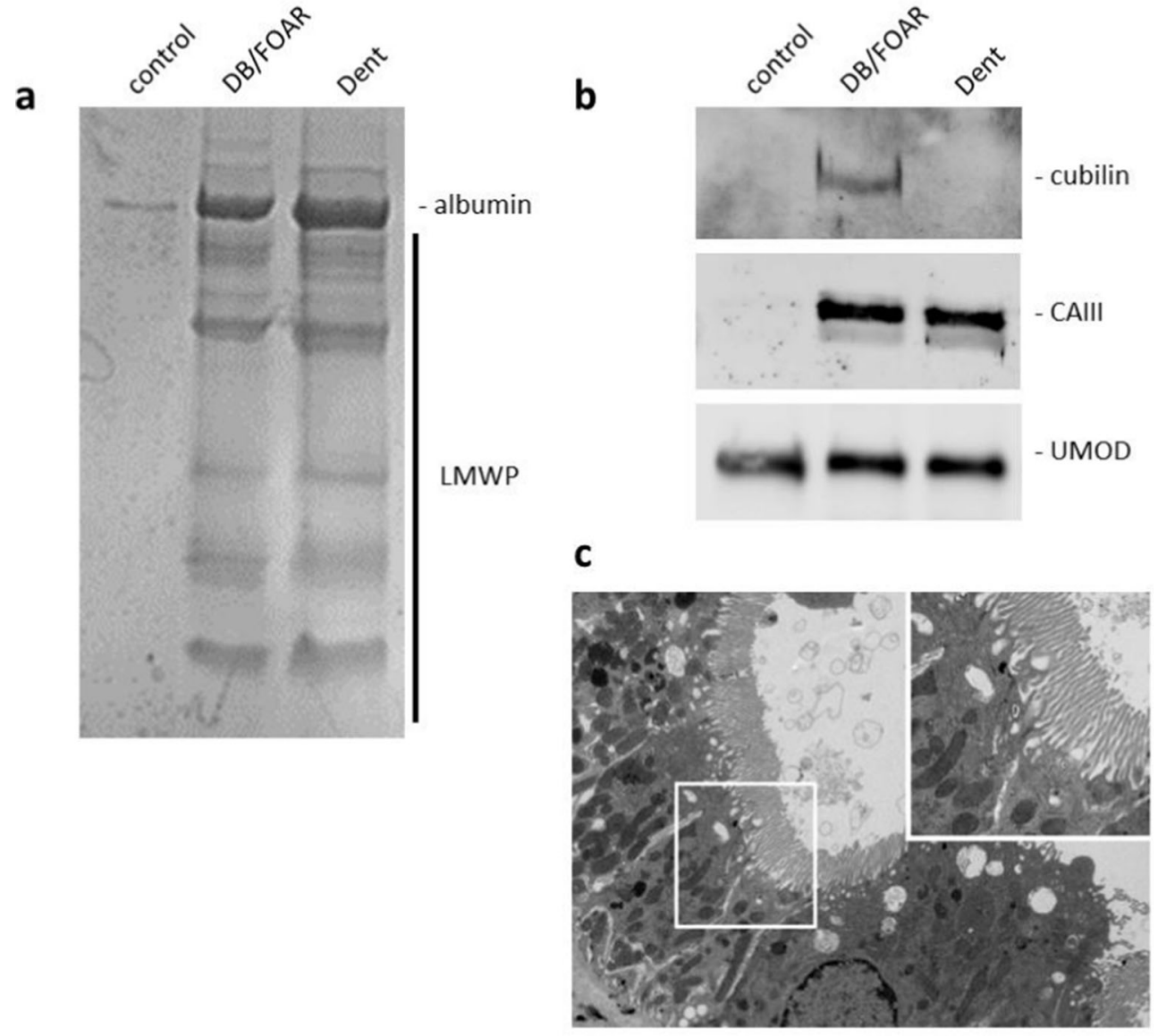

d

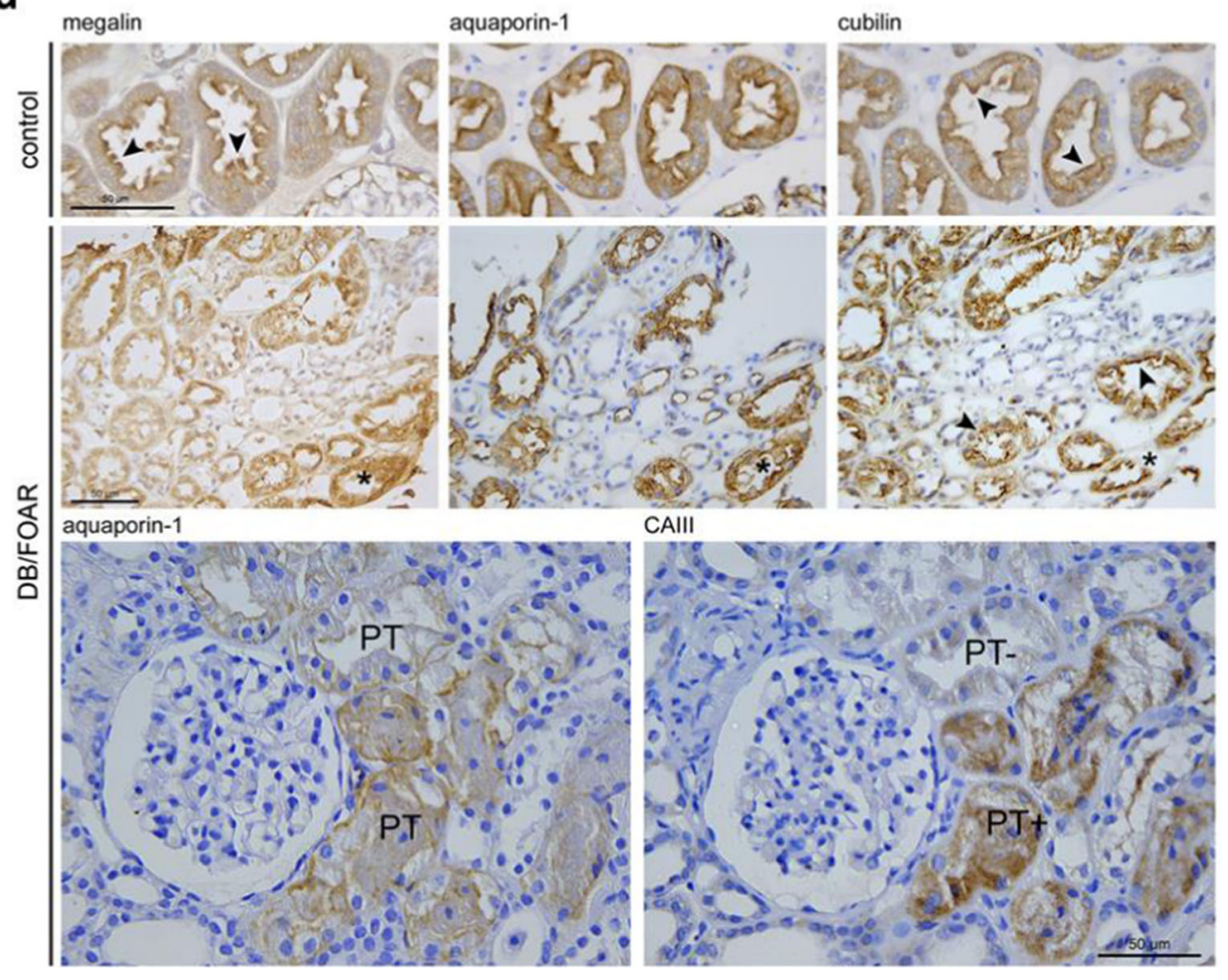


proteinuria [2]. The amount of physiological glomerular filtration of albumin remains debated [3]. In mice, PT uptake of filtered albumin relies on the co-expression of megalin and cubilin [1]. Consistently, patients with DB/ FOAR show daily albuminuria, like in our case $(\sim 250 \mathrm{mg} / 24 \mathrm{~h})$. Since no PT reabsorption of albumin is observed in these individuals, physiological albumin filtration may be extrapolated to the microalbuminuric rather than nephrotic range. Similarly to previous reports $[2,3]$, both megalin ligands, i.e., $\alpha 1$-microglobulin, RBP and NGAL, and cubilin ligands, i.e., transferrin and cystatin, are found in our patient's urine. By immunostaining of kidney samples, megalin was detectable as a weak and diffuse signal in PT cytosol, which suggests that C.7564T $>\mathrm{C}$ and c. $12623 \mathrm{C}>\mathrm{A}$ mutations of $L R P 2$ induce a trafficking defect and/or instability of megalin. By contrast, cubilin distribution was preserved at the PT brush border, indicating that cubilin processing and targeting are independent of megalin function [1]. However, cubilin was also found in the patient's urine, in contrast with age- and gender-matched healthy controls and a patient with Dent's disease. This supports that, in case of megalin dysfunction, cubilin is shed into the urine, which in turn leads to urinary loss of cubilin-specific ligands. Urinalysis also detected CAIII. In normal physiological conditions, mouse and human urine is devoid of CAIII. Conversely, its abundance is significantly augmented in kidney and urine samples from ClC-5-deficient mice and patients [8]. Here, we show that CAIII is detectable in kidney and urine samples from a DB/ FOAR patient, which suggests that CAIII may represent a biomarker of PT dysfunction beyond Dent's disease. As compared with traditional biomarkers, like $\alpha_{1}$ - and $\beta_{2}$-microglobulin, which are produced outside the kidney, filtered but not reabsorbed because of defective RME, CAIII in the urine may directly reflect a state of PT cell injury, without changes in renal function or morphology. Additional investigations are required to substantiate the relevance of CAIII in other types of inherited or acquired renal Fanconi syndrome.

The C.7564T > C mutation was described in the original report associating DB/FOAR to LRP2 mutations [2]. It concerns an extracellular LDL-receptor class B motif. The c. $12623 \mathrm{C}>\mathrm{A}$ mutation is novel, and affects a proline residue located in another extracellular LDL-receptor class B motif of megalin, with a substitution by histidine. The proline residue at position 4208 of megalin is highly conserved throughout evolution, as suggested by comparative sequence alignment using Alamut. The physicochemical gap between proline and histidine is significant, with a Grantham distance of 77. Classical genomic databases, such as Polyphen, predict that c.12623C > A mutation pathologically perturbs $\mathrm{pH}$-dependent ligand/ megalin dissociation in endosomes. Finally, we found an impaired development of apical endocytic apparatus and a reduced and mislocalized expression of megalin in PT cells, which further underscores the pathogeny of both LRP2 mutations.

Long-term evolution of kidney function in DB/FOAR patients remains unknown. Twenty-seven cases from 15 families have been reported thus far. Ten reports concern deceased in utero or neonatal cases. Among the other cases, two girls developed renal insufficiency at the age of 18-20 [3,9]. The rareness of DB/FOAR hampers efforts to assess long-term prognosis. Also, the variability in phenotype severity might be influenced by the type of $L R P 2$ mutations. On a pathophysiological note, a decrease in megalin membrane expression has been associated with altered survival in cultured PT cells [10]. Furthermore, dysfunction in PT RME leads to protein overload in the distal nephron, which mediates inflammation and secretion of profibrotic factors [11]. Still, the long-term impact on renal function in the numerous forms of inherited renal Fanconi syndrome understandably depends on the mutated genes and implicated pathways.

Besides the kidney, megalin is expressed in many absorptive epithelia, including the neuro-epithelium. There, megalin is thought to participate to RME-associated cell signaling. Failure to uptake ligands at a specific time and/ or in a tissue-specific manner during fetal development may provoke the various clinical abnormalities observed in DB/FOAR. Hence, during brain development, megalin mediates neural tubule specification by acting as a clearance receptor of various ligands, such as bonemorphogenic protein 4 , from extra-embryonic fluids [12]. In megalin-deficient mice, impaired ligand uptake by neuro-epithelial cells ultimately leads to holoprosencephaly, i.e., fusion of prosencephalic hemispheres. During optic nerve development, megalin modulates sonic hedgehog abundance, thereby favoring the recruitment of oligodendrocyte precursors [13]. In adult mouse eyes, megalin is expressed in vesicular structures of the retinal pigment epithelium (RPE) and nonpigmented ciliary body epithelium. Genetic ablation of megalin in mouse causes myopia, which is associated with enlarged RPE melanosomes and abnormal ciliary body development [14]. As a whole, these observations further support that megalin harbors important roles in brain and eye development and physiology.

In conclusion, the present clinical, biochemical, and genetic investigations of a girl with DB/FOAR provide new insights into this rare clinical entity and help further understand the pathophysiology of the PT endocytic pathway. It not only reports on a novel missense mutation of $L R P 2$ gene causing DB/FOAR, but also highlights the role of megalin in apical RME and cubilin membrane stability in PT cells. 
Acknowledgments The authors are thankful to all members of the Department of Pediatrics at CHC Hospital for their help and helpful suggestions about this clinical case, as well as to L. Poma for skillful technical assistance. Comments and suggestions of E. Castermans (Division of Genetics, Ulg CHU, Liège, Belgium) and O. Hougrand (Department of Pathology, Ulg CHU, Liège, Belgium) were greatly appreciated. FJ is an MD Postdoctoral Fellow of the Fonds National de la Recherche Scientifique (FNRS) in the unit GIGA Cardiovascular Sciences (Prof. JM Krzesinski and J-O Defraigne) of the University of Liège, and receives support from the FNRS (Research Credit 3309), the University of Liège (Fonds Spéciaux à la Recherche) and from the Fonds Léon Fredericq. The present study was performed after approval by the Ethics Committee of the University of Liège Hospital.

Conflict of interest None.

\section{References}

1. Christensen EI, Birn H, Storm T, Weyer K, Nielsen R (2012) Endocytic receptors in the renal proximal tubule. Physiology (Bethesda) 27:223-236

2. Kantarci S, Al-Gazali L, Hill RS, Donnai D, Black GC, Bieth E, Chassaing N, Lacombe D, Devriendt K, Teebi A, Loscertales M, Robson C, Liu T, MacLaughlin DT, Noonan KM, Russell MK, Walsh CA, Donahoe PK, Pober BR (2007) Mutations in LRP2, which encodes the multiligand receptor megalin, cause DonnaiBarrow and facio-oculo-acoustico-renal syndromes. Nat Genet 39: 957-959

3. Storm T, Tranebjaerg L, Frykholm C, Birn H, Verroust PJ, Neveus T, Sundelin B, Hertz JM, Holmstrom G, Ericson K, Christensen EI, Nielsen R (2013) Renal phenotypic investigations of megalindeficient patients: novel insights into tubular proteinuria and albumin filtration. Nephrol Dial Transplant 28:585-591

4. Leheste JR, Rolinski B, Vorum H, Hilpert J, Nykjaer A, Jacobsen C, Aucouturier P, Moskaug JO, Otto A, Christensen EI, Willnow TE (1999) Megalin knockout mice as an animal model of low molecular weight proteinuria. Am J Pathol 155:1361-1370
5. Devuyst O, Jouret F, Auzanneau C, Courtoy PJ (2005) Chloride channels and endocytosis: new insights from Dent's disease and ClC-5 knockout mice. Nephron Physiol 99:p69-p73

6. Jouret F, Devuyst O (2009) CFTR and defective endocytosis: new insights in the renal phenotype of cystic fibrosis. Pflugers Arch 457: $1227-1236$

7. Christensen EI, Devuyst O, Dom G, Nielsen R, Van der Smissen P, Verroust P, Leruth M, Guggino WB, Courtoy PJ (2003) Loss of chloride channel ClC-5 impairs endocytosis by defective trafficking of megalin and cubilin in kidney proximal tubules. Proc Natl Acad Sci U S A 100:8472-8477

8. Gailly P, Jouret F, Martin D, Debaix H, Parreira KS, Nishita T, Blanchard A, Antignac C, Willnow TE, Courtoy PJ, Scheinman SJ, Christensen EI, Devuyst O (2008) A novel renal carbonic anhydrase type III plays a role in proximal tubule dysfunction. Kidney Int 74 : 52-61

9. Pober BR, Longoni M, Noonan KM (2009) A review of DonnaiBarrow and facio-oculo-acoustico-renal (DB/FOAR) syndrome: clinical features and differential diagnosis. Birth Defects Res A Clin Mol Teratol 85:76-81

10. Caruso-Neves C, Pinheiro AA, Cai H, Souza-Menezes J, Guggino WB (2006) PKB and megalin determine the survival or death of renal proximal tubule cells. Proc Natl Acad Sci U S A 103:18810-18815

11. Mahadevappa R, Nielsen R, Christensen EI, Birn H (2014) Megalin in acute kidney injury: foe and friend. Am J Physiol Renal Physiol 306:F147-F154

12. Spoelgen R, Hammes A, Anzenberger U, Zechner D, Andersen OM, Jerchow B, Willnow TE (2005) LRP2/megalin is required for patterning of the ventral telencephalon. Development 132:405-414

13. Ortega MC, Cases O, Merchan P, Kozyraki R, Clemente D, de Castro F (2012) Megalin mediates the influence of sonic hedgehog on oligodendrocyte precursor cell migration and proliferation during development. Glia 60:851-866

14. Storm T, Heegaard S, Christensen EI, Nielsen R (2014) Megalindeficiency causes high myopia, retinal pigment epitheliummacromelanosomes and abnormal development of the ciliary body in mice. Cell Tissue Res 358:99-107

15. Jouret F, Igarashi T, Gofflot F, Wilson PD, Karet FE, Thakker RV, Devuyst O (2004) Comparative ontogeny, processing, and segmental distribution of the renal chloride channel, ClC-5. Kidney Int 65:198-208 